\title{
Reunião Internacional sobre Vigilância e Prevenção da Doença de Chagas na Amazônia. Implementação da Iniciativa Intergovernamental de Vigilância e Prevenção da doença de Chagas na Amazônia
}

\author{
Manaus, Estado de Amazonas, Brasil, 19 a 22 de setembro de 2004
}

Os Delegados Oficiais dos países amazônicos, e um painel de pesquisadores e técnicos, especialistas em diversos aspectos da pesquisa, da prevenção e do manejo da doença de Chagas, reunidos no Centro de Pesquisa Leônidas e Maria Deane FIOCRUZ Amazônia, com a Secretaria Técnica da Organização Pan-Americana da Saúde ( OPAS) , e o patrocínio e apoio da Chagas' Disease Intervention Activities-European Community (CDIA-EC), elaboraram um grupo de recomendações e diretrizes, agrupadas em quatro propostas temáticas:

A) Avaliação dos riscos de estabelecimento da endemia chagásica na Amazônia.

B) Pesquisa necessária para a vigilância e prevenção da doença de Chagas na Amazônia.

C) Proposta de medidas de vigilância e prevenção da doença de Chagas.

D) Proposta de um sistema de cooperação internacional para a vigilância e prevenção da doença de Chagas na Amazônia ( AMCHA) .

A) Avaliação dos riscos de estabelecimento da endemia chagásica na Amazônia

Na maioria dos países da bacia amazônica tem sido relatada a infecção por Trypanosoma cruzi em seres humanos, reservatórios animais, triatomíneos e casos de doença de Chagas.

Embora Carlos Chagas, em seu momento histórico, tenha descrito a presença da parasitose em reservatórios na Amazônia, até agora o problema tem recebido pouca atenção e não é relatado habitualmente pelos serviços de saúde. 0 conhecimento da ocorrência da doença ainda é limitado, mas algumas observações e evidências mostram que o problema pode ser muito mais grave do que se pensa.

Situações de transmissão

1. Transmissão em zonas rurais e peri-urbanas com vetores silvestres
Essa transmissão está relacionada com Rhodnius robustus e R pictipes, que invadem os domicílios sem colonizá-los, e Panstrongylus geniculatus, que tem sido encontrado em peridomicílios. Isso traz um alerta acerca do potencial de transmissão da espécie.

Na Amazônia equatoriana e no Rio Negro, no Brasil, têm sido observados perfis epidemiológicos que mostram risco cumulativo em relação à idade, assim como transmissão contínua já faz algumas décadas. Situações similares no caso de outros vetores têm sido relatadas no Peru e na Colômbia, sendo possível que fenômenos parecidos ocorram em outras áreas de distribuição desses vetores.

2. Triatomíneos que colonizam de forma incipiente os domicílios

2.1 A situação de T. maculata.

T. maculata, reconhecido na Venezuela como um vetor secundário de importância na área extra-amazônica, está sendo capturado com frequiência em habitações humanas no Estado de Roraima, no Brasil.

2.2 P. herreri e R. ecuadoriensis no Peru.

P. herreri e R. ecuadoriensis são os principais vetor de localização intradomiciliar e intradomiciliar, respectivamente. Ambos têm sido encontrados infectados por T. cruzi eT. rangeli.

\section{Situações particulares de transmissão}

\subsection{Surtos focais}

Tem sido relatada a ocorrência de surtos focais provavelmente associados com a transmissão pela via oral, especialmente com suco de açaí (Euterpe catinga). Não se encontrou outra evidência satisfatória.

Acredita-se que triatomíneos infectados e/ou suas fezes contaminam alguma fase do processamento e consumo do produto, e que essa seria a via de transmissão. Até 1998, tinham sido descritos 17 episódios envolvendo 85 casos, sendo que a maioria ocorreu no Estado do Pará e os outros, no Amapá, Acre e Amazonas mais recentemente.

Essa transmissão pelos alimentos abriria uma nova linha de pesquisa e cooperação técnica com 0 INPPAZ ( Instituto Pan-Americano de Proteção de Alimentos e Zoonoses) da OPAS. 


\subsection{Extração da fibra de piaçava (Leopoldina piassaba)}

No Norte do Rio Negro, no Brasil, tem sido documentada a transmissão de T cruzi relacionada com a atividade de extração das fibras da palmeira L piassaba, afetando às famílias que entram no ecotopo natural do vetor, $0 \mathrm{R}$ brethesi.

\subsection{Práticas culturais}

Na Colômbia é comum a ingestão de sangue de tatus e gambás como remédio na medicina tradicional de alguns grupos indígenas na Amazônia, o que representa um risco de transmissão de T cruzi. 0 mesmo papel poderia ter a ingestão de carne de reservatórios pouco cozida ou apenas defumada em outros grupos na região amazônica.

\section{Atividades que podem aumentar o risco de transmissão}

- Destruição da floresta causada por:

crescimento urbano;

estabelecimento de grandes áreas de pasto;

aumento das plantações de açaí e pupunha,

substituindo a floresta primária;

atividades extrativistas, como a coleta de castanhas na Bolívia.

- Aumento expressivo e repentino das populações humanas.

- Grupos de trabalho em atividades de mineração e outras, sem medidas adequadas de proteção.

- Turismo na floresta sem as devidas medidas de proteção.

\section{Riscos do ambiente domiciliar}

- Casas construídas na floresta, cercadas por árvores, incluindo palmeiras.

- Pessoas com alto risco de contato com triatomíneos

- Condições propícias à presença de vetores, reservatórios domésticos e silvestres nas casas

- Habitações construídas com materiais naturais dos ecotopos dos triatomíneos.

- A luz dos domicílios abertos, servindo de isca para atrair os triatomíneos.

\section{Perfil epidemiológico}

- As formas de exposição ao risco de contato com triatomíneos são muito variadas na Amazônia.

- É provável que a ausência de observações relativas à domiciliação dos triatomíneos na Amazônia tenha sugerido a idéia de que a endemicidade chagásica não constitui um problema para a região.
- A transmissão pode ocorrer em diferentes níveis de intensidade, relacionados com as distintas atividades humanas.

- 0 modo de transmissão vetorial clássico conhecido em outras zonas endêmicas, consistente na transmissão dependente da domiciliação, parece não ter se estabelecido de maneira significativa na Amazônia. Esse fato pode ter retardado a compreensão epidemiológica da doença de Chagas nessa região.

- As informações epidemiológicas acerca da endemia são insuficientes para se conhecer a verdadeira situação. Conseqüentemente, ainda não é possível propor um conjunto completo de medidas de prevenção e controle.

- A ausência de dados sobre a endemia na Amazônia está relacionada com a capacidade exígua de vigilância epidemiológica e entomológica dos serviços de saúde na região.

\section{Necessidades de pesquisa}

- Fortalecer e refinar as capacidades de diagnóstico clínico e laboratorial, determinando o significado de resultados cruzados e definindo um protocolo de diagnóstico sorológico.

- Determinar quais reservatórios poderiam estar implicados na transmissão de T. cruzi na Amazônia.

- Ampliar os estudos soroepidemiológicos em áreas peri-urbanas e zonas com transmissão comprovada.

- Comprovar a via de transmissão nos surtos focais anteriormente descritos.

- Conhecer as características clínicas da doença de Chagas na Amazônia.

\section{B) Pesquisa necessária à vigilância e prevenção da doença de Chagas na Amazônia}

Em termos gerais, o grupo entende que tem havido progresso no conhecimento da doença de Chagas na Amazônia ao longo dos últimos dois anos. As informações disponíveis, acrescidas dos recentes relatórios dos Países apresentados nesta Reunião, reforçam 0 consenso que se firma entre os estudiosos quanto aos dois principais pressupostos do presente workshop:

- A tripanossomíase americana ocorre com alta prevalência e ampla dispersão como enzootia silvestre em toda a Amazônia, apresentando elementos favoráveis a sua expansão como endemia humana;

- Aimplantação de um sistema de vigilância e prevenção da doença de Chagas humana é considerada muito oportuna no presente momento, em que se detectam 
casos humanos agudos e crônicos em praticamente todos os Países da região, crescem a ação antrópica e 0 crescimento demográfico na área, e são detectados inúmeros vetores e reservatórios naturalmente infectados nas vastas extensões pesquisadas.

Mesmo assim, 0 grupo reconhece que esta tripanossomíase apresenta características próprias e muito particulares na Amazônia, que a diferenciam, substancialmente, dos padrões habituais da doença de Chagas que tem sido descrita e estudada principalmente no Cone Sul. Da mesma forma, reconhece-se a falta de uma longa série de informações sobre diversos aspectos da entidade, sendo ainda desconhecidos, por exemplo, desde aspectos elementares da bio-ecologia das populações dos vetores e parasitas envolvidos como detalhes e extensão da doença humana até as formas de transmissão na área. De permeio, grandes dificuldades operacionais e de locomoção, a miscigenação de agravos e múltiplos elementos antigênicos, 0 ambiente mutante $\mathrm{e}$ vários fatores geopolíticos complicam sobremaneira a realização de um diagnóstico claro da situação e tornam intempestiva e arriscada a implantação sumária de medidas tradicionais de controle. Por tudo isso, o componente de pesquisa deve ser considerado prioritário e imprescindível na gestão da Iniciativa que este workshop está propondo aos países integrantes da região.

Nesse sentido, o quadro básico da investigação proposta definese, fundamentalmente, pelo caráter aplicado e de exeqüibilidade em relação aos diversos aspectos envolvidos na vigilância e prevenção da doença de Chagas humana na Amazônia, esperandose que a mesma seja comum a todos os países da Iniciativa. Em termos de sustentação, o grupo vê como natural e pertinente que este componente de pesquisa faça parte da agenda e dos orçamentos regulares dos Governos participantes, cabendo também prioridade junto às Agências Internacionais, como TDR, JICA, CE e outras.

Como forma de trabalho, 0 Grupo procurou relacionar as prioridades de linhas e cenários de risco, a partir de intensas discussões acerca dos principais componentes da história natural da tripanossomíase já identificados ou sugeridos em pesquisas anteriores na área, assim como das necessidades e dos problemas sentidos por vários pesquisadores presentes em relação à pesquisa da doença de Chagas na Amazônia. Os tópicos indicados a seguir foram abordados e genericamente discriminados em termos de necessidades e propostas de investigação. É evidente que essa relação possui natureza meramente introdutória no presente documento, devendo ser complementada e aprimorada, ao longo da Iniciativa, pelos diferentes grupos de pesquisa envolvidos. Pela ordem, seguem-se os resumos das discussões e as propostas relativas a cada item.

\section{1) Vetores}

Apesar dos avanços significativos, ainda há muito a pesquisar nesse tema. Indagações genéricas sobre quais são os vetores existentes e quais, os principais vetores na transmissão da infecção humana, qual é a distribuição das espécies e quais são os padrões gerais de sua distribuição nos ambientes e paisagens. Em geral, aceita-se que a imensa maioria dos vetores na Amazônia são silvestres e têm pouca tendência à domiciliação em ecótopos artificiais, 0 que pode sofrer modificações e deve ser monitorado pela vigilância. Reconhecem-se diversas e múltiplas variedades de microclimas e micro-ambientes na Amazônia como um todo, sendo ainda pouco conhecidos os fatores de dispersão e contenção da maioria das espécies registradas. Em termos práticos para a Iniciativa, se propõe duas atividades de imediato, sendo:

a) Importante levantar, nos países da região, quais são a capacidade e as competências existentes para coletar e identificar as espécies ocorrentes, assim como estudálas em profundidade quanto aos diversos aspectos de vigilância e prevenção da doença de Chagas;

b) Importante que a Iniciativa estimule e apóie a elaboração e publicação, por especialistas designados, de uma lista atualizada dos vetores já encontrados na Amazônia, com os dados disponíveis de dispersão, taxas de infestação domiciliar e colonização e infecção natural por T. cruzi, ecótopos de detecção etc., como base para revisões e atualizações regulares, no futuro, observando-se, é claro, as tendências e mudanças ocorridas.

№ curto e meio prazos, é importante que os países disponham de Laboratórios de Entomologia de referência nacional, integrados em rede, entre si e com centros de excelência na região e no exterior, nos moldes da Rede "ECLAT" ", aliás uma das entidades precursoras desta e de outras iniciativas de controle da doença de Chagas.

Em termos de vigilância e coleta regular, a exemplo das propostas da Bolívia, da Colômbia e do Equador, os agentes dos programas nacionais e regionais de malária poderiam participar das ações de busca ativa domiciliar, além de fomentar e viabilizar a detecção de triatomíneos pela população das áreas.

Nesse sentido é muito importante identificar os fatores e as circunstâncias ecológicas, culturais, sociais e ambientais que facultam a interação do homem com os triatomíneos nas diversas paisagens amazônicas, procurando, inclusive, elucidar situações próprias e específicas, como a transmissão da doença humana por triatomíneos invasores que não colonizam (Equador) e surtos familiares ou vicinais de transmissão oral, sendo provável que os triatomíneos sejam a origem do parasita transmitido (Brasil). 
Já no âmbito da pesquisa sobre as espécies e os ambiente silvestres, o grupo reconhece sua enorme importância e as numerosas dificuldades inerentes a essa investigação, devendo a mesma ser empreendida por grupos e projetos especializados, dando prioridade às áreas próximas ou vinculadas a assentamentos humanos, ainda mais em situações de ocorrência da transmissão da doença e/ou detecção de domiciliação e invasão. Em geral, deve-se priorizar a pesquisa em palmeiras ( habitat preferencial do gênero mais importante na Amazônia (Rhodnius), de preferência através de sua dissecação ou, alternativamente, pelo emprego de armadilhas já existentes ou que venham a ser desenvolvidas ${ }^{2}$.

Seria ideal dispor de um protocolo mínimo para essas investigações, em termos de amostragem, estratégias, indicadores e métodos, o que asseguraria a comparabilidade entre diferentes estudos.

Como temas mais pontuais de investigação identificados neste item, assinalaram-se ainda (lista que deve ser oportunamente revista e ampliada) as seguintes linhas de pesquisa.

- Estudos específicos sobre as espécies R. prolixus e R. robustus, objetivando melhor compreensão da interação de uma com a outra, seu potencial de hibridização na natureza, eventual potencial de R. robustus de se domiciliar, facilidades e possibilidades de identificação taxonômica ${ }^{3}$, etc.

- Estudos específicos sobre a expansão de P. geniculatus predomiciliado em áreas do Pará ( Brasil) e outros países da região.

- Estudos comparativos da morfologia e capacidade vetorial de P. herreri e P. lignarius em suas diferentes áreas de dispersão, sabendo-se que conceituados autores as consideram como uma única espécie;

- Estudos comparativos e analíticos sobre R. ecuadoriensis no Equador (basicamente silvestres) e no Peru (sempre domiciliados);

- Estudos específicos sobre a distribuição por espécie, a partir de microclimas, para os níveis "macro", consolidando as informações e visando a prever a dispersão;

- Apoiar e continuar os estudos de entomologia planejados pelo Programa Boliviano através dos laboratórios regionais e agentes de controle da malária.

- Estudar a possibilidade de implantação de "unidades sentinelas" para estudo e monitoramento de triatomíneos em regiões estratégicas;

- Estudar as culturas, os costumes, os hábitos e as atitudes das populações nativas, especialmente indígenas, em relação aos triatomíneos;
- Estudar a influência das ações de controle químico dos programas de controle da malária nos triatomíneos da Amazônia, em termos tanto de sua repelência como da eventual potenciação da resistência aos praguicidas empregados no controle dessa doença;

- Otimizar o levantamento de triatomíneos em áreas adjacentes a focos detectados da doença humana;

- Considerar a hipótese de se realizar sorologia em seres humanos nas localidades e/ou unidades domiciliares onde se encontrarem triatomíneos invasores ou domiciliados;

- Estudar comparativamente e em seu conjunto os padrões físicos das unidades domiciliares encontradas colonizadas, buscando definir fatores de risco;

- Atenção especial e monitoramento de regiões de migração e assentamentos, visando à possível introdução passiva de espécies alóctones.

\section{2) Reservatórios}

Os reservatórios também são tema de grande importância, visto as especificidades e a grande dispersão das populações de T. cruzi até agora detectadas na Amazônia. É extensa a relação das espécies encontradas infectadas naturalmente, sendo que a última revisão geral dessa relação foi realizada por Mauro Barretto (Brasil) nos anos 1960. Por isto, existe consenso de que a Iniciativa deveria estimular a atualização dessa relação, como base para revisões periódicas futuras. Conforme apontado anteriormente, a Iniciativa também deveria elaborar protocolos padronizados de pesquisa, visando à comparabilidade dos achados.

A prospeção básica deveria ser realizada usando métodos parasitológicos convencionais (xenodiagnóstico e hemocultura), acrescidos de técnicas moleculares e de anatomo-patologia, quando disponíveis.

As pesquisas de laboratório podem ajudar a melhorar a compreensão da circulação do parasita pela via oral, entre reservatórios silvestres e, eventualmente, no homem.

Atenção especial deve ser dada a marsupiais e roedores silvestres, por suas características sinantrópicas. No caso dos marsupiais, seria interessante pesquisar 0 ciclo particular do T. cruzi nas glândulas anais.

É importante promover parcerias dos pesquisadores com grupos especializados em ecologia e sistemática das espécies envolvidas. Em particular, sugere-se que os países membros procurem identificar centros de referência em taxonomia zoológica, aproveitando as oportunidades de cooperação nacional e internacional.

\footnotetext{
2. Nesse ponto, a associação e cooperação dos entomologistas, ecologistas e especialistas em palmeiras mostra-se muito importante e já tem tido excelentes resultados práticos nas várias experiências relatadas.

3. Nesse sentido, o Instituto Oswaldo Cruz desenvolveu uma técnica de identificação/separação molecular dessas espécies, que prometeu disponibilizar aos laboratórios da Região.
} 
№ âmbito doméstico, o grupo acredita ser particularmente importante a investigação sistemática da infecção por T. cruzi em cães e gatos que vivem em volta das habitações humanas e podem ser contaminados pelo parasita por diferentes vias, especialmente a oral, ingerindo triatomíneos ou roedores infectados. Além disso, deveria se estudar, em áreas de circulação do parasita, a mobilidade de espécies como Rattus rattus entre as habitações humanas e 0 ambiente silvestre.

\section{3) 0 parasita}

Reconhece-se a complexidade e dificuldade de estudo deste tópico na Amazônia, de modo geral. Existe um grande leque de populações, especialmente nas áreas silvestres, e, aparentemente, têm sido isolados e caracterizados na região apenas indivíduos das linhagens I e III (classificação do Consenso de 1999, Memórias do IOC 94, supl. 1). Seria ideal se esses trabalhos pudessem ser realizados integralmente na própria região, em laboratórios de excelência com capacidade para isolar e caracterizar bioquimica e molecularmente os parasitas. Em especial, circulam, na Amazônia, muitos outros flagelados similares a T. cruzi, como T. saimirii, T. rangeli e Phytomonas sp, entre outros, o que não só complica a identificação desses organismos usando microscopia comum, como ainda confunde os resultados de sorologia em seres humanos e mamíferos reservatórios.

No caso da fase aguda em seres humanos, a pesquisa clássica usando técnicas parasitológicas diretas e indiretas constitui a metodologia de excelência e deveria ser implementada nos laboratórios de diagnóstico. Para laboratórios de referência, que dispõem de protocolos atualizados da FIOCRUZ, recomenda-se a implantação da metodologia de PCR, cuja finalidade básica é aumentar a especificidade dos diagnósticos, fato importante principalmente na Amazônia. A técnica também serve para auxiliar na tipagem por grupo, que é mais complicada e implica maiores exigências de qualidade técnica, mas é importante, especialmente nos estudos relativos à Amazônia.

Quanto à diferenciação com T. rangeli, a técnica molecular está disponível, sugerindo-se sua implantação nos laboratórios na região Amazônica. Para otimizar a identificação em reservatórios e triatomíneos, 0 melhor seria isolar e cultivar 0 parasita, 0 que nem sempre é muito fácil, especialmente com cepas do grupo I.

Todos esses estudos são muito importantes no campo vigilância epidemiológica e serão fundamentais para mapear as tendências da infecção por T. cruzi na região. Também interessa a caracterização do parasita em pacientes crônicos, o que deve ser feito a partir de isolamentos. Isso tem se mostrado bastante difícil em alguns estudos na Amazônia. Finalmente, é importante a manutenção de bancos de cepas da região, o que pode se viabilizar de maneira simples em alguns dos laboratórios de referência.

\section{4) Diagnóstico de casos crônicos humanos}

Rotineiramente, no Cone Sul e em outras regiões, 0 diagnóstico é efetuado mediante sorologia convencional, a qual apresenta problemas de sensibilidade e, especialmente, de especificidade na Amazônia. Seria a ferramenta básica dos estudos soro-epidemiológicos, mas deve ser revista e considerada com cautela na Região Amazônica, devido, principalmente, ao grande número de falsos positivos ocorrido em diversos levantamentos. Nas pesquisas do Alto Rio Negro (Brasil), a caracterização final dos soros é realizada, habitualmente, usando 0 Western Blot, pois duas técnicas clássicas convencionais não são suficientes. Esse assunto deveria ser pesquisado em profundidade na Amazônia, por meio de um estudo específico de sorologia na área, envolvendo vários laboratórios. Em paralelo, 0 grupo sugere pesquisas objetivando o aperfeiçoamento de métodos moleculares para 0 diagnóstico complementar de casos humanos na Amazônia.

\section{5) Clínica e infecção humana}

Aparentemente, a doença de Chagas humana aguda é similar na Amazônia e no Cone Sul no tocante à parasitemia e às taxas de mortalidade, embora existam diferenças no perfil clínico. Sugere-se um protocolo padronizado de avaliação e caracterização clínicas desses casos, tanto os detectados em pacientes febris isolados ( lâminas de malária) como aqueles detectados em episódios familiares.

Em particular, seria muito interessante que a via de transmissão ficasse esclarecida e caracterizada em detalhe em todos esses casos, abrindo espaço para ações de vigilância melhores e mais efetivas.

Para os casos agudos, em termos de prevenção secundária, é imperioso iniciar o tratamento especíicico, sendo muito convenientefazer estudos longitudinais de seguimento clínico e laboratorial, a posteriori. Em particular, reitera-se a recomendação de seminários anteriores (Santarém 2001, Palmari 2002) no sentido de rede de laboratórios de diagnóstico demalária participar ativamente da pesquisa de casosagudos de doença de Chagas através da capacitação (e supervisão sistemática) dos laboratoristas de campo que realizam hemoscopia.

Para os casos crônicos, além da procura de um melhor diagnóstico etiológico, deveriam ser desenvolvidos protocolos de seguimento visando à melhor caracterização das formas clínicas, inclusive procurando identificar quais são os quadros clínicos mais característicos da Região.

Como linhas de investigação complementar nos casos crônicos, sugeriu-se pesquisa regular e aprofundada em bancos de sangue e nos serviços de verificação de óbitos, padronizada em toda a Região.

Entre as sugestões de pesquisa pontual adicional estão a investigação e implementação da vigilância nos municípios com maior incidência de episódios familiares no Estado do Pará, no Brasil, e a investigação experimental com PCR em pacientes não-agudos com sorologia convencional negativa.

Ainda como recomendação prática adicional, sugere-se, ao TDR (e/ou aos Ministérios da Saúde ou Ciência e Tecnologia dos Países da Região) , a possibilidade de viabilizar um programa de estímulo à pesquisa para projetos pontuais, do tipo "pequenas bolsas de pesquisa" a ser gerido por comitê próprio da Iniciativa, visando aos temas prioritários da vigilância e prevenção da doença de Chagas na Amazônia. 


\section{C) Proposta de medidas de vigilância e prevenção da doença de Chagas}

\section{Introdução}

Énecessário enfatizar que existem elementos, estruturas, objetivos e perspectivas comuns a todos os países relativos ao desenvolvimento da vigilância da doença de Chagas na Sub-região Amazônica.

A proposta fundamenta-se na conservação da identidade do sistema de vigilância de cada país, em termos de sua organização e seu funcionamento, assim como de seu sistema de saúde e sua gestão ambiental nacional.

\section{Definição}

Propõe-se a implementação de um sistema de vigilância integrada da doença de Chagas que empregue os sistemas de vigilância de doenças transmitidas por vetores e vigilância epidemiológica já existentes nos países, com um desenvolvimento progressivo visando à intersetorialidade e boa articulação com as estruturas nacionais de Atenção Primaria de Saúde (APS), procurando assegurar sua eficácia, eficiência, sensibilidade, oportunidade de ação e sustentabilidade.

\section{Objetivos}

Os objetivos propostos são:

- detectar a infecção humana por T. cruzi,

- detectar as situações de risco devido a contato dos seres humanos com os triatomíneos e

- detectar situações de risco, em função de fatores:

- sociais,

- étnicos,

- econômicos,

- produtivos e

- culturais,

- entre outros possíveis.

\section{0pções de Estrutura}

A) Sistema mínimo de vigilância

- baseado em definições operacionais preestabelecidas;

- integrado aos sistemas de vigilância já existentes na Sub-región: malária, doenças emergentes e atenção primaria de saúde;

- com componente de vigilância passiva associado à malária (lâminas) e às doenças emergentes ( sorologia) ;
- com componente de vigilância ativa: levantamentos sorológicos e pesquisa focal em situações de risco;

- desenvolvimento de um sistema de notificação, pelas comunidades, às autoridades sanitárias, da presença de triatomíneos em âmbitos domiciliares ou em diversas situações de risco por contato vetor-homen, com especial ênfase no uso do sistema de ensino básico; e

- com componente de controle e tratamento conseqüentes.

\section{B) Sistema de vigilância máxima, com integração intersetorial e extra-setorial}

- meio ambiente,

- educação,

- agricultura,

- organizações da sociedade civil,

- organizações étnicas e

- projetos produtivos,

- entre outros.

\section{Componentes}

1. Vigilância epidemiológica, baseada em um sistema de informação, diagnóstico, tratamento, promoção da saúde, participação comunitária e informação, educação e comunicação em saúde (IEC).

2. Vigilância ambiental que inclua:

- vigilância entomológica,

- qualidade ambiental ( clima, contaminação, etc.),

- saneamento ambiental (habitação, peridomicílio, etc.) e

- ecossistemas (reservatórios, vegetação) .

3. Desenvolvimento de um componente de investigação com retroalimentação ( feedback) pesquisa «vigilância e vigilância«investigação.

4. Inserção das ações de controle da doença de Chagas na APS.

5. Capacitação de recursos humanos pertencentes a sistemas em funcionamento e à APS nacional em diagnóstico e entomologia, basicamente:

- microscopistas que trabalham com malária e dos serviços de saúde,

- auxiliares de entomologia do sistema de saúde,

- técnicos ambientalistas de diversos sistemas e

- profissionais de APS. 
O sistema deverá operar com um ponto focal nacional por país ( Delegado Oficial na Comissão Intergovernamental da Iniciativa, com sua instituição de apoio) e um ponto focal subregional (Grupo Técnico-Executivo da Iniciativa), que deverão elaborar um relatório anual das atividades, por país e sub-región, no formato que for acordado. Esse relatório deverá ser apresentado na reunião anual da Iniciativa.

Todo mês, os programas nacionais, por meio de seus pontos focais, encaminharão suas informações via Internet, a endereço eletrônico a ser definido.

Observe-se que a implementação do sistema pressupõe ( para os países e outros integrantes) um investimento mínimo em capacitação e equipamento básico a serem financiados sem a criação de estruturas paralelas.

A presente proposta está sujeita a avaliações periódicas, de maneira a efetuar ajustes e reengenharia dos processos propostos em função da experiência operacional acumulada.

\section{D) Proposta de um sistema de cooperação internacional para vigilância e prevenção da doença de Chagas na Amazônia (AMCHA)}

Os delegados dos países da Região Amazônica, participantes da Reunião Internacional sobre Vigilância e Prevenção da doença de Chagas, propõem a criação de una "Iniciativa dos países amazônicos para a vigilância e o controle da doença de Chagas" (AMCHA), a ser formalmente constituída por decisão e vontade política dos Ministros da Saúde de Bolívia, Brasil, Colômbia, Equador, França, Guiana, Peru, Suriname e Venezuela.

\section{0 objetivo general da Iniciativa AMCHA é:}

Prevenir 0 estabelecimento em ampla escala da transmissão vetorial endêmica da doença de Chagas na região amazônica através dos seguintes objetivos específicos:

- implantar/implementar um sistema de vigilância epidemiológica regular, integrado e com aproveitamento máximo dos recursos, serviços e programas de saúde já existentes;

- desenvolver métodos e/ou técnicas de controle ajustados aos mecanismos de transmissão já conhecidos ( transmissão vetorial extradomiciliar, transmissão domiciliar sem colonização pelo vetor, transmissão oral) ;

- gerar novos conhecimentos acerca da epidemiologia da doença na região ( segundo as pautas de investigação definidas no item B)

Para a obtenção desses objetivos e operacionalização dessas atividades propõe-se como estrutura de apoio a constituição de: i) una Comissão Intergovernamental (CI) a ser constituída por expressa e formal delegação das autoridades sanitárias dos países quando a proposta aqui explicitada for homologada pelos Ministros da Saúde;

ii) uma Secretaria Técnica, a cargo da OPAS/OMS, por meio de sua Representação no Brasil; e

iii) um Grupo de Coordenação Técnica-Executiva com atribuições específicas e sede rotativa de dois anos, definidas pelos delegados dos países membros. Amissão desse grupo de coordenação técnica será a de articular os esforços nacionais para dar cumprimento às recomendações e aos mandatos da CI. Seria conveniente, em vista das necessidades de pesquisa da Região, que fossem incorporados, como assessores da Comissão de Coordenação Técnica-Executiva, dois membros representativos da comunidade científica para supervisionar, estimular e coordenar as investigações em andamento.

Nesta Reunião determinou-se que o Brasil será o primeiro país-sede do grupo de Coordenação Técnica-Executiva da Iniciativa, cuja tarefa imediata será propor o estabelecimento e a padronização dos procedimentos da vigilância epidemiológica. Essa proposta deverá ser discutida numa próxima reunião ad-hoc com pessoal técnico especializado indicado por cada país membro.

A responsabilidade do grupo de Coordenação TécnicaExecutiva estará a cargo de uma instituição a ser indicada pelo Governo do país-sede, com base em competências e requisitos a serem definidos pela Secretaria Técnica da Iniciativa.

Para a operacionalização das atividades da Iniciativa propõe-se:

1. Reunião anual da CI, em sedes rotativas, para analisar os avanços, as realizações e as limitações do processo de implementação da Iniciativa, assim como estabelecer as metas anuais a serem cumpridas.

2. Utilizar os projetos de cooperação técnica entre dois ou mais países (TCCs/OPAS), principalmente nesta fase inicial, considerando que o grau de acumulação de conhecimentos é muito diverso.

3. Com base nas parcerias já existentes, que podem continuar trazendo benefícios para a Iniciativa, criar foros de intercâmbio entre os países e as instituições associadas ou que venham a se associar à Iniciativa, tais como CDIA/ ECLAT, OTCA, Médicos sem Fronteiras, Cooperação Européia.

Reconhecendo que esta Reunião não possui atribuições para criar a Iniciativa, propõe-se que, para concretizar formalmente esta necessidade, se faça 'advocacia' para que os Ministros da Saúde dos nove países que compartilham a Bacia Amazônica e a Guiana Francesa acordem a criação da Iniciativa no âmbito da 
próxima Reunião de Ministros da Saúde da América do sul, a ser realizada em março de 2005, na cidade de Santiago de Chile, ou antes, se houver uma oportunidade. Isso significa que é necessário apresentar a devida justificativa aos respetivos governos, para 0 qual se acorda a implementação das seguintes ações:

- solicitar que a OTCA inclua a Iniciativa AMCHA em sua Comissão Especial de Saúde Pública (CESAM) e que tome para si os objetivos da mesma,

- solicitar que a OPASdissemine e divulgue o presente documento
- solicitar que o ORAS-CONHU e as Representaç̃es da OPAS nos países desenvolvam atividades de 'advocacia' junto aos Ministros da Saúde para que aceitem a inclusão da proposta de criação da Iniciativa na Agenda da próxima reunião de Ministros da Saúde ( Santiago de Chile, 2005) .

- Os Delegados dos Países presentes a esta reunião levarão, a suas respectivas autoridades, a proposta de criação da Iniciativa como recomendação da presente reunião.

Manaus, Estado de Amazonas, Brasil, 22 de setembro de 2004

Dr. Amadeo Rojas (Bolívia), Dr. Márcio Vinhães (Brasil), Dr. Mauricio Rodríguez (Colômbia), Dr. Jorge Monroy (Equador), Dr. Navindra Persaud (Guiana*), Dra. Christine Aznar (Guiana Francesa / França), Dr. César Náquira (Peru), Mrs. Helene Hiwat (Suriname)

e Dr. Jesús Benítez (Venezuela*)

*Ausência Justificada 